\title{
THE WIENER-PITT PHENOMENON ON THE HALF-LINE
}

\author{
by J. H. WILLIAMSON \\ (Received 11th September, 1961)
}

IT has been well known for many years (2) that if $F_{\mu}(t)$ is the Fourier-Stieltjes transform of a bounded measure $\mu$ on the real line $R$, which is bounded away from zero, it does not follow that $\left[F_{\mu}(t)\right]^{-1}$ is also the Fourier-Stieltjes transform of a measure. It seems of interest (as was remarked, in conversation, by J. D. Weston) to consider measures on the half-line $R^{+}=[0, \infty[$, instead of on $R$. The Fourier-Stieltjes transform is now replaced by the LaplaceStieltjes transform

$$
L_{\mu}(\zeta)=\int_{0}^{\infty} e^{-x \zeta} d \mu(x) \quad(\mathscr{R} \zeta \geqq 0),
$$

and the problem is: if $\mu$ is a bounded measure, and

$$
\left|L_{\mu}(\zeta)\right| \geqq k>0 \quad(\mathscr{R} \zeta \geqq 0)
$$

is it true that $\left[L_{\mu}(\xi)\right]^{-1}$ is the Laplace-Stieltjes transform of a measure also? The answer, as will be shown below, is negative; the Wiener-Pitt phenomenon occurs. One may of course ask (and to some extent answer) a similar question in the case of a general semigroup. Some extensions (for example, to the positive quadrant in $R^{2}$, and similar situations) are immediate; we do not attempt to discuss the general problem here.

The occurrence of the phenomenon in the case of $R^{+}$follows quite easily from results already known for $R$. Let $M(R)$ be the Banach algebra of bounded measures on $R$, and let $\lambda \in M(R)$ satisfy

(i) $\|\lambda\|=1$;

(ii) the support of $\lambda$ is contained in $[-1,1]$;

(iii) $F_{\lambda}(t)$ is real for all $t$;

(iv) the spectrum of $\lambda$ contains $i\left(i^{2}=-1\right)$.

It is clear that (i) and (iii) together imply that $-1 \leqq F_{\lambda}(t) \leqq 1$ for all $t$. The existence of such measures has been established in general locally compact abelian groups (see (1) or (4)). A simple example on the real line may be obtained (3) by writing $\lambda_{n}$ for the measure with mass $\frac{1}{2}$ at each of $\pm 1 / n$ !, and taking $\lambda$ to be the infinite convolution product $\lambda_{2 *} \lambda_{3} \lambda_{4} \ldots$. Let $\mu$ be the measure $\delta_{3 *}\left(\delta_{0}-\lambda^{2}\right)$, that is, the measure obtained by translating $\left(\delta_{0}-\lambda^{2}\right)$ through a distance +3 (we write $\delta_{x}$ for the measure with mass 1 at $x$ ). Then $\mu$ may be regarded as a measure on either $R$ or $R^{+}$; its support is contained in $(1,5)$. 
Let $m$ be a homomorphism of $M(R)$ on to the complex field such that $m\left(\lambda^{2}\right)=-1$; let $m\left(\delta_{3}\right)=e^{i \theta}$, and consider the measure

Then

$$
\nu=2 \delta_{0}-e^{-i \theta} \mu \text {. }
$$

$$
m(v)=2-e^{-i \theta} e^{i \theta}(1+1)=0,
$$

and so $v$ has no inverse in $M(R)$ and, a fortiori, no inverse in $M\left(R^{+}\right)$.

On the other hand, $L_{v}(\zeta)$ is bounded away from zero in the right half plane. Writing $\zeta=\xi+i \eta$ we have in general, for $\xi \geqq 0$,

$$
\begin{aligned}
\left|L_{v}(\zeta)-2\right| & =\left|\int_{0}^{\infty} e^{-x \zeta} d \mu(x)\right| \\
& =\left|\int_{1}^{5} e^{-x \zeta} d \mu(x)\right| \\
& \leqq\left|e^{-\zeta}\right|\|\mu\| \\
& =2 e^{-\xi}
\end{aligned}
$$

so that $L_{v}(\zeta)$ is bounded away from zero in every half plane $\xi \geqq \xi_{0}>0$. Also, for $\xi=0$,

$$
L_{v}(i \eta)=F_{v}(\eta)=2-e^{-i \theta} e^{-3 i \eta}\left(1-F_{\lambda}^{2}(\eta)\right) .
$$

Since $0 \leqq F_{\lambda}^{2}(\eta) \leqq 1$, it follows that $\left|L_{v}(i \eta)\right| \geqq 1$. Finally, $L_{v}(\zeta)$ is continuous in $\xi$, uniformly in $\eta$, at $\xi=0$ (and indeed everywhere). Taking $\xi>0$ we have

$$
\begin{aligned}
\left|L_{v}(i \eta)-L_{v}(\xi+i \eta)\right| & =\left|\int_{0}^{\infty}\left(1-e^{-x \xi}\right) e^{-i x \eta} d v(x)\right| \\
& =\left|\int_{0}^{5}\left(1-e^{-x \xi}\right) e^{-i x \eta} d v(x)\right| \\
& \leqq\|v\| \sup _{0 \leqq x \leqq 5}\left(1-e^{-x \xi}\right) \\
& =4\left(1-e^{-5 \xi}\right) .
\end{aligned}
$$

From this, the required result follows immediately.

Since if $\left[L_{v}(\xi)\right]^{-1}$ were of the form $L_{\sigma}(\zeta)$ for some bounded measure $\sigma$, it would follow that $\sigma=v^{-1}$, it is clear that the Wiener-Pitt phenomenon occurs.

\section{REFERENCES}

(1) W. RudIN, Bull. American Math. Soc., 65 (1959), 227-247.

(2) N. Wiener and H. R. Pitt, Duke Math. J., 4 (1938), $420-436$.

(3) J. H. Williamson, Communication at International Congress of Mathematicians (Edinburgh, 1958).

(4) J. H. Williamson, Proc. Edin. Math. Soc., 11 (1959), 195-206.

King's COLlege

CAMBridge 